\title{
Fundamental Properties of Fuzzy Implications
}

\author{
Adam Grabowski \\ Institute of Informatics \\ University of Białystok \\ Poland
}

\begin{abstract}
Summary. In the article we continue in the Mizar system [8, 2] the formalization of fuzzy implications according to the monograph of Baczyński and Jayaram "Fuzzy Implications" 1. We develop a framework of Mizar attributes allowing us for a smooth proving of basic properties of these fuzzy connectives [9]. We also give a set of theorems about the ordering of nine fundamental implications: Łukasiewicz $\left(I_{\mathrm{LK}}\right)$, Gödel $\left(I_{\mathrm{GD}}\right)$, Reichenbach $\left(I_{\mathrm{RC}}\right)$, Kleene-Dienes $\left(I_{\mathrm{KD}}\right)$, Goguen $\left(I_{\mathrm{GG}}\right)$, Rescher $\left(I_{\mathrm{RS}}\right)$, Yager $\left(I_{\mathrm{YG}}\right)$, Weber $\left(I_{\mathrm{WB}}\right)$, and Fodor $\left(I_{\mathrm{FD}}\right)$.

This work is a continuation of the development of fuzzy sets in Mizar [6]; it could be used to give a variety of more general operations on fuzzy sets 13 . The formalization follows [10], [5], and [4].
\end{abstract}

MSC: 03B52 68T37 03B35

Keywords: fuzzy implication; fuzzy set; fuzzy logic

MML identifier: FUZIMPL2, version: 8.1.08 5.53.1335

\section{INTRODUCTION}

There are two fundamental aims of this Mizar article: first of all, I wanted to introduce in the Mizar Mathematical Library how nine basic fuzzy implications formally defined in [4] are ordered - and this result is given in Section 2 as a formal counterpart of Example 1.1.6, p. 3 of [1].

On the other hand, in the final section I prove the formal characterization of fundamental fuzzy implications in terms of four elementary properties [12] expressed in Table 1.4 of [1], p. 10 (note the absence of the continuity of the operators in our version of this presentation). Here 
- (NP) - the left neutrality property,

- $(\mathrm{EP})$ - the exchange principle,

- (IP) - the identity principle,

- $(\mathrm{OP})$ - the ordering property.

Actually, this is the part of Example 1.3.2, p. 9 from [1]:

\begin{tabular}{|c|c|c|c|c|}
\hline Fuzzy implication & $(\mathrm{NP})$ & $(\mathrm{EP})$ & $(\mathrm{IP})$ & $(\mathrm{OP})$ \\
\hline$I_{\mathrm{LK}}$ & + & + & + & + \\
\hline$I_{\mathrm{GD}}$ & + & + & + & + \\
\hline$I_{\mathrm{RC}}$ & + & + & - & - \\
\hline$I_{\mathrm{KD}}$ & + & + & - & - \\
\hline$I_{\mathrm{GG}}$ & + & + & + & + \\
\hline$I_{\mathrm{RS}}$ & - & - & + & + \\
\hline$I_{\mathrm{YG}}$ & + & + & - & - \\
\hline$I_{\mathrm{WB}}$ & + & + & + & - \\
\hline$I_{\mathrm{FD}}$ & + & + & + & + \\
\hline \multicolumn{4}{|r}{}
\end{tabular}

Additionally, Section 4 contains registrations of clusters of adjectives allowing for further work in more automated framework within fuzzy sets [3] - this is the Mizar version of Lemma 1.3.3 and 1.3.4 from [1]. Such automatization can be especially useful in the hybridization of fuzzy and rough approaches [7].

\section{Preliminaries}

We introduce the notation $I_{\mathrm{LK}}$ as a synonym of the Eukasiewicz implication and $I_{\mathrm{GD}}$ as a synonym of the Gödel implication. We introduce $I_{\mathrm{RC}}$ as a synonym of the Reichenbach implication and $I_{\mathrm{KD}}$ as a synonym of the Kleene-Dienes implication.

We introduce $I_{\mathrm{GG}}$ as a synonym of the Goguen implication and $I_{\mathrm{RS}}$ as a synonym of the Rescher implication. We introduce $I_{\mathrm{YG}}$ as a synonym of the Yager implication and $I_{\mathrm{WB}}$ as a synonym of the Weber implication and $I_{\mathrm{FD}}$ as a synonym of the Fodor implication.

From now on $x, y$ denote elements of $[0,1]$. Now we state the propositions:

(1) $\left.\square^{1}=(\operatorname{AffineMap}(1,0)) \Gamma\right] 0,+\infty[$.

Proof: Set $f=\square^{1}$. Set $\left.g=(\operatorname{AffineMap}(1,0)) \uparrow\right] 0,+\infty[$. For every object $x$ such that $x \in \operatorname{dom} f$ holds $f(x)=g(x)$.

(2) Let us consider real numbers $a, b$. Then 
(i) AffineMap $(a, b)$ is differentiable on $\mathbb{R}$, and

(ii) for every real number $x,(\operatorname{AffineMap}(a, b))^{\prime}(x)=a$.

(3) If $0<x<1$ and $0<y<1$, then $\left.\left(\square^{x}+(\operatorname{AffineMap}(-x, x-1))\right) \Gamma\right] 0,1[$ is increasing.

Proof: Set $f_{1}=\square^{x}$. Set $f_{2}=\operatorname{AffineMap}(-x, x-1)$. Reconsider $\left.Y=\right] 0,1[$ as an open subset of $\mathbb{R}$. Set $f=f_{1}+f_{2}$. Set $\left.A=\right] 0,+\infty\left[. f_{2}\right.$ is differentiable on $A$. $f_{1} \uparrow A$ is differentiable on $A$. $f_{2}$ is differentiable on $Y$. For every real number $y$ such that $y \in Y$ holds $0<f^{\prime}(y)$ by [11, (21)], (2).

(4) Let us consider a real number $u$. Suppose $u \in] 0,1]$.

Then $\left(\square^{x}+(\operatorname{AffineMap}(-x, x-1))\right)(u)=u^{x}-1+x-x \cdot u$.

\section{The Ordering of Fuzzy Implications}

Now we state the propositions:

(5) (i) if $x \leqslant y$, then $\left(I_{\mathrm{LK}}\right)(x, y)=1$, and

(ii) if $x>y$, then $\left(I_{\mathrm{LK}}\right)(x, y)=1-x+y$.

(6) (i) if $x=0$, then $\left(I_{\mathrm{GG}}\right)(x, y)=1$, and

(ii) if $x>0$, then $\left(I_{\mathrm{GG}}\right)(x, y)=\min \left(1, \frac{y}{x}\right)$.

(7) $I_{\mathrm{KD}} \leqslant I_{\mathrm{RC}} \leqslant I_{\mathrm{LK}} \leqslant I_{\mathrm{WB}}$.

(8) $I_{\mathrm{RS}} \leqslant I_{\mathrm{GD}} \leqslant I_{\mathrm{GG}} \leqslant I_{\mathrm{LK}} \leqslant I_{\mathrm{WB}}$.

(9) $I_{\mathrm{RC}} \leqslant I_{\mathrm{LK}} \leqslant I_{\mathrm{WB}}$.

(10) $I_{\mathrm{KD}} \leqslant I_{\mathrm{FD}} \leqslant I_{\mathrm{LK}} \leqslant I_{\mathrm{WB}}$.

(11) $I_{\mathrm{RS}} \leqslant I_{\mathrm{GD}} \leqslant I_{\mathrm{FD}} \leqslant I_{\mathrm{LK}} \leqslant I_{\mathrm{WB}}$.

\section{Additional Properties of Fuzzy Implications}

Let $I$ be a binary operation on $[0,1]$. We say that $I$ satisfies $(\mathrm{NP})$ if and only if

(Def. 1) for every element $y$ of $[0,1], I(1, y)=y$.

We say that $I$ satisfies (EP) if and only if

(Def. 2) for every elements $x, y, z$ of $[0,1], I(x, I(y, z))=I(y, I(x, z))$.

We say that $I$ satisfies (IP) if and only if

(Def. 3) for every element $x$ of $[0,1], I(x, x)=1$.

We say that $I$ satisfies (OP) if and only if

(Def. 4) for every elements $x, y$ of $[0,1], I(x, y)=1$ iff $x \leqslant y$. 
In the sequel $I$ denotes a binary operation on $[0,1]$.

Let $I$ be a binary operation on $[0,1]$. We introduce the notation $I$ satisfies $(\mathrm{NC})$ as a synonym of $I$ is 01 -dominant and $I$ satisfies (I1) as a synonym of $I$ is antitone w.r.t. 1st coordinate.

We introduce $I$ satisfies (I2) as a synonym of $I$ is isotone w.r.t. 2nd coordinate and $I$ satisfies (I3) as a synonym of $I$ is 00 -dominant and $I$ satisfies (I4) as a synonym of $I$ is 11-dominant and $I$ satisfies (I5) as a synonym of $I$ is 10 -weak.

\section{Dependencies Between Chosen Properties}

Now we state the proposition:

(12) If $I$ satisfies (LB), then $I$ satisfies (I3) and (NC).

One can verify that every binary operation on $[0,1]$ which satisfies (LB) satisfies also (I3) and (NC).

Now we state the proposition:

(13) If $I$ satisfies (RB), then $I$ satisfies (I4) and (NC).

One can check that every binary operation on $[0,1]$ which satisfies $(\mathrm{RB})$ satisfies also (I4) and (NC).

Now we state the proposition:

(14) If $I$ satisfies (NP), then $I$ satisfies (I4) and (I5).

Note that every binary operation on $[0,1]$ which satisfies (NP) satisfies also (I4) and (I5).

Now we state the proposition:

(15) If $I$ satisfies (IP), then $I$ satisfies (I3) and (I4).

Let us note that every binary operation on $[0,1]$ which satisfies (IP) satisfies also (I3) and (I4).

Now we state the proposition:

(16) If $I$ satisfies (OP), then $I$ satisfies (I3), (I4), (NC), (LB), (RB), and (IP).

One can verify that every binary operation on $[0,1]$ which satisfies $(\mathrm{OP})$ satisfies also (I3), (I4), (NC), (LB), (RB), and (IP).

Now we state the proposition:

(17) If $I$ satisfies (EP) and (OP), then $I$ satisfies (I1), (I3), (I4), (I5), (LB), $(\mathrm{RB}),(\mathrm{NC}),(\mathrm{NP})$, and (IP).

One can verify that every binary operation on $[0,1]$ which satisfies (EP) and (OP) satisfies also (I1), (I5), and (NP). 


\section{Properties of Nine Classical Fuzzy Implications}

Let us note that $I_{\mathrm{LK}}$ satisfies (NP), (EP), (IP), and (OP).

$I_{\mathrm{GD}}$ satisfies $(\mathrm{NP}),(\mathrm{EP}),(\mathrm{IP})$, and $(\mathrm{OP})$.

$I_{\mathrm{RC}}$ satisfies $(\mathrm{NP})$ and $(\mathrm{EP})$ but does not satisfy (IP) and (OP).

$I_{\mathrm{KD}}$ satisfies $(\mathrm{NP})$ and $(\mathrm{EP})$ but does not satisfy $(\mathrm{IP})$ and $(\mathrm{OP})$.

$I_{\mathrm{GG}}$ satisfies $(\mathrm{NP}),(\mathrm{EP}),(\mathrm{IP})$, and $(\mathrm{OP})$.

Let us note that $I_{\mathrm{RS}}$ satisfies (IP) and (OP) but does not satisfy (NP) and $(\mathrm{EP})$.

$I_{\mathrm{YG}}$ satisfies (NP) and (EP) but does not satisfy (IP) and (OP).

$I_{\mathrm{WB}}$ satisfies (NP), (EP), and (IP) but does not satisfy (OP).

$I_{\mathrm{FD}}$ satisfies (NP), (EP), (IP), and (OP).

$I_{0}$ satisfies (EP) but does not satisfy (NP), (IP), and (OP).

$I_{1}$ satisfies (EP) and (IP) but does not satisfy (NP) and (OP).

\section{REFERENCES}

[1] Michał Baczyński and Balasubramaniam Jayaram. Fuzzy Implications. Springer Publishing Company, Incorporated, 2008. doi 10.1007/978-3-540-69082-5.

[2] Grzegorz Bancerek, Czesław Byliński, Adam Grabowski, Artur Korniłowicz, Roman Matuszewski, Adam Naumowicz, and Karol Pąk. The role of the Mizar Mathematical Library for interactive proof development in Mizar. Journal of Automated Reasoning, 61(1):9-32, 2018. do1 $10.1007 / \mathrm{s} 10817-017-9440-6$

[3] Didier Dubois and Henri Prade. Fuzzy Sets and Systems: Theory and Applications. Academic Press, New York, 1980.

[4] Adam Grabowski. Formal introduction to fuzzy implications. Formalized Mathematics, 25(3):241-248, 2017. doi 10.1515/forma-2017-0023.

[5] Adam Grabowski. Basic formal properties of triangular norms and conorms. Formalized Mathematics, 25(2):93-100, 2017. doi 10.1515/forma-2017-0009

[6] Adam Grabowski. On the computer certification of fuzzy numbers. In M. Ganzha, L. Maciaszek, and M. Paprzycki, editors, 2013 Federated Conference on Computer Science and Information Systems (FedCSIS), Federated Conference on Computer Science and Information Systems, pages 51-54, 2013.

[7] Adam Grabowski and Takashi Mitsuishi. Initial comparison of formal approaches to fuzzy and rough sets. In Leszek Rutkowski, Marcin Korytkowski, Rafal Scherer, Ryszard Tadeusiewicz, Lotti A. Zadeh, and Jacek M. Zurada, editors, Artificial Intelligence and Soft Computing - 14th International Conference, ICAISC 2015, Zakopane, Poland, June 14-18, 2015, Proceedings, Part I, volume 9119 of Lecture Notes in Computer Science, pages 160-171. Springer, 2015. doi:10.1007/978-3-319-19324-3_15.

[8] Adam Grabowski, Artur Korniłowicz, and Adam Naumowicz. Four decades of Mizar. Journal of Automated Reasoning, 55(3):191-198, 2015. doi:10.1007/s10817-015-9345-1

[9] Petr Hájek. Metamathematics of Fuzzy Logic. Dordrecht: Kluwer, 1998.

[10] Takashi Mitsuishi, Noboru Endou, and Yasunari Shidama. The concept of fuzzy set and membership function and basic properties of fuzzy set operation Formalızed Mathematıcs, 9(2):351-356, 2001.

[11] Yasunari Shidama. The Taylor expansions Formalized Mathematics, 12(2):195-200, 2004.

[12] Philippe Smets and Paul Magrez. Implication in fuzzy logic. International Journal of Approximate Reasoning, 1(4):327-347, 1987. doi $10.1016 / 0888-613 \mathrm{X}(87) 90023-5$. 
[13] Lotfi Zadeh. Fuzzy sets. Information and Control, 8(3):338-353, 1965. doi:10.1016/S00199958(65)90241-X

Accepted September 29, 2018 